\title{
THE IMPORTANCE OF UPPER FIRST PERMANENT MOLARS POSITION FOR THE ORTHOGNATIC OCCLUSION
}

\author{
Gergana Ivanova, Hristina Arnautska, Zornitsa Vulcheva, Iliana Atanasova \\ Department of Orthodontics, Faculty of Dental Medicine, Medical University of Varna
}

\begin{abstract}
Development of the dental arches and occlusion in permanent dentition can be divided into several stages and has to be observed regularly. The first permanent molar eruption is related to the onset of significant changes in the developing occlusion. Although this tooth is seen as the "key to occlusion" its value as an anchorage is debatable.

The aim of the article is to study the correct position of the upper first molars in the two planes of space the sagittal and transverse planes.

In this article the position of the first upper molar is examined with the aid of diagnostic records, such as study cast, orthopantomogram (OPG), and lateral cephalometrics. A literature review includes Bulgarian and foreigner authors.

Angle, who in 1899 referred to the maxillary first permanent molars as the "key to occlusion", was the first to mention their importance within the dentition. According to Angle, the line passing through the middle of the mesiobuccal cusp of the upper first molar coincides with the line passing through the buccal groove of the lower first molar. After Angle, other authors have discussed the position of upper molars from different point of view, such as their relation or position in the maxilla, anteroposterior axial inclination and rotation. As indicated by Lamons and Holmes molar rotations commonly exist in Class II malocclusions. The molars are usually rotated around an axis lingual to their central fossae. In an ideal occlusion the buccal surfaces of the upper first molars are usually parallel to each other

On the OPG Kurol and Bjerklin measured the axial mesial inclination of upper first molar. The tipping of the molars is measured by the angle formed between the tangent line to the mesial surfaces of the root and crown and the line through the lower margins of the left and right orbits.
\end{abstract}

According to Sassouni, the mesial contour of upper first molar should to lie on the 4th arc - the temporal arc. If the molar is anterior to this arc, a treatment with distalization could be initiated. The temporal arcnasion distance measured on the radius is equal to the distance from point ANS to the upper first molar. The position of the upper first molar varies with the position of the upper central incisors. The basic hypothesis is that if the upper first molar has a fixed position in the face, any increase in the total upper dental arch

Address for correspondence:

Gergana Ivanova

Faculty of Dental Medicine

Medical University of Varna

84 Tzar Osvoboditel Blvd

9000 Varna

e-mail: gergana-ivanova@hotmail.com

Received: December 3, 2017

Accepted: December 29, 2017 length will be transferred to the incisor area. Any change in the anteroposterior position of the upper first molar could influence the position of the mandibular- leading to Class II malocclusion.

Ricketts pointed out that the average distance from the pterygoid vertical (PTV) to the distal surface of upper first molar is the sum of the age of the patient 
The Importance of Upper First Permanent Molars Position for the Orthognatic Occlusion

$+3 \mathrm{~mm}$, in a growing patient. This diagnostic method can help the orthodontist to decide whether to extract teeth or to distalize the molars.

Any loss of space in the arch is a justification for early orthodontic treatment. Mediopalatal rotation of the upper molar is an additional problem in the final phase as well. The rotation of upper first molars is measured by the angles formed by the intersection of lines going over the tips of the mesiopalatal and distobuccal cusps of each molar (Ricketts line) with a straight line marked over the palatine raphe.

The problem of reduced arch length has an impact on the final treatment stage when the major orthodontic goal is establishing a tight teeth intercuspation. The molars influence the transfer of occlusal forces to the facial skeleton. The upper first molar tolerates more changes in the position than the lower one. The correct position of the upper molar ensures a stable occlusion with significantly low grade of relapse.

Keywords: upper first molar, key to occlusion, rotation, orthognatic occlusion

\section{INTRODUCTION}

Development of the dental arches and occlusion in permanent dentition can be divided into several stages and has to be observed regularly. The first permanent molar eruption is related to the onset of significant changes in the developing occlusion. Although this tooth is seen as the "key to occlusion" its value as an anchorage is debatable.

\section{AIM}

The aim of the article is to study the correct position of the upper molars in the two planes of space - the saggital and transverse planes.

\section{MATERIALS AND METHODS}

Based on almost 200 articles, a literature review was conducted to determine the correct position of upper first permanent molar with the aid of diagnostic records including study casts, orthopantomogram (OPG), and lateral cephalographs.

\section{DISCUSSION}

The intramaxillary position of the molar has received little attention in orthodontic literature (1).

Angle, who in 1899 consider the maxillary first permanent molars the "key to occlusion", was the first to note their importance for occlusion (2). According to Angle, the line passing through the middle of the mesiobuccal cusp of the upper first molar coincides with the line passing through the buccal groove of the lower first molar (Fig. 1). The maxillary first permanent molar has been the tooth most commonly used as a key in classifying dentition in terms occlusion. If these molars are not in their ideal position, they may lead to a malrelationship (3).

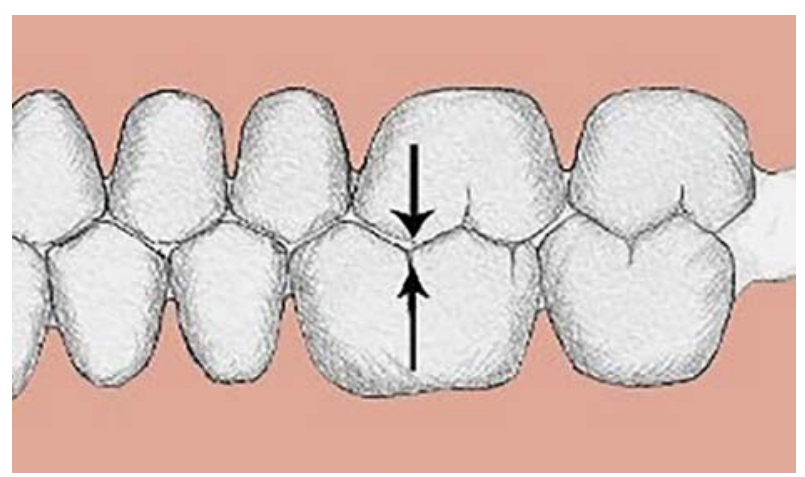

Fig. 1. The correct interdigitation between the molars Class I

This classification of Angle was supplemented by the "Six Keys to Normal Occlusion" by Andrews. Three of the keys are really important for the correct position of the upper first permanent molars. The first of the six keys is molar relationship. The distal surface of the distobuccal cusp of the upper first permanent molar occludes the mesial surface of the mesiobuccal cusp of the lower second molar. The second key is the crown angulation - the crown tip is expressed in degrees, plus or minus. The degree of the crown tip is the angle between the long axis of the crown (as viewed from the labial or buccal surface) and a line bearing 90 degrees from the occlusal plane. A plus reading is awarded when the gingival portion of the long axis of the crown is distal to the incisal portion. A minus reading is assigned when 
Gergana Ivanova, Hristina Arnautska, Zornitsa Vulcheva et al.

the gingival portion of the long axis of the crown is mesial to the incisal portion (Fig. 2).

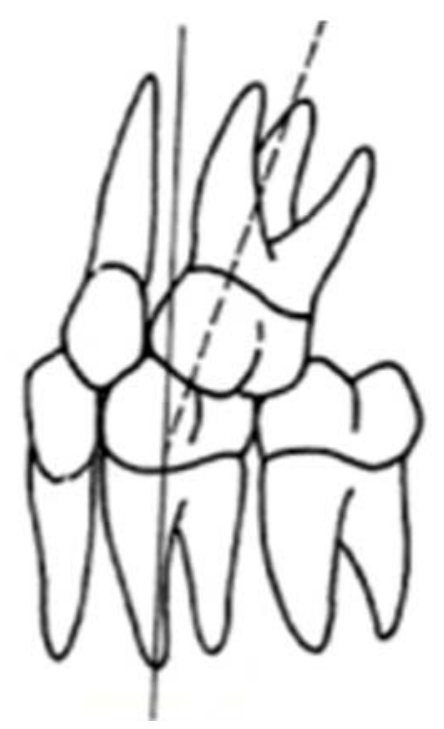

Fig. 2. Proper molar relationship (1 $1^{\text {st }}$ key of Andrews)

The fourth key to normal occlusion is that the teeth should be free of undesirable rotations. The molar, if rotated, would occupy more space than normal, creating a situation unreceptive to normal occlusion (4).

Stoller (3) corrected the misconception that the mesiobuccal cusp of the upper first permanent molar occludes in the buccal groove of the lower first permanent molar in normal occlusion, when viewed buccally. He wrote: "The position of the maxillary first molar in normal occlusion was noted to be such that its mesiobuccal cusp occluded distally to the buccal groove of the mandibular first molar."

Strang (5) said: "The buccal ridge of the mesiobuccal lobe of the first molar stands out so far buccally that it gives the arch form a distinct offset buccally as it passes from the second premolar to the first molar area."

After Angle, other authors have discussed the position of upper molars from different viewpoints, such as their relation or position in the maxilla, anteroposterior axial inclination and rotation. An abnormal rotation of molars has a definite harmful effect on the occlusion, even if small in actual dimensions (6). According to Hellmans the abnormal rotation of the maxillary first permanent molars leads to confusion in classification (7). All types of malocclusion exhibit rotation of the molar as a general rule.

As indicated by Lamons and Holmes (8), molar rotations commonly exist in Class II malocclusions. The molars are usually rotated around an axis lingual to their central fossae (9). When the upper first molar drifts mesially, the large lingual root contacts the lingual cortical bone plate and allows the two buccal roots to rotate mesiolingually (10). The lingual root act as the center or axis of rotation and the two buccal roots may rotate in a segment of a circle without breaking through the buccal plate of bone. The rhomboidal shape of the maxillary first molar occupies a greater amount of space in the arch and contributes to the relapse of distocclussion (11).

Henry (12) is one of the first authors to present a method of measuring the degree of rotation. In order to discover the degree of rotation he used the angle formed by a line joining the points of the mesiobuccal and distobuccal cusps and the median raphe (Fig. 3). The standard magnitude of the angle is 10 degrees \pm 4 degrees. Above 14 degrees, the molar is considered to have a mesiolingual rotation.

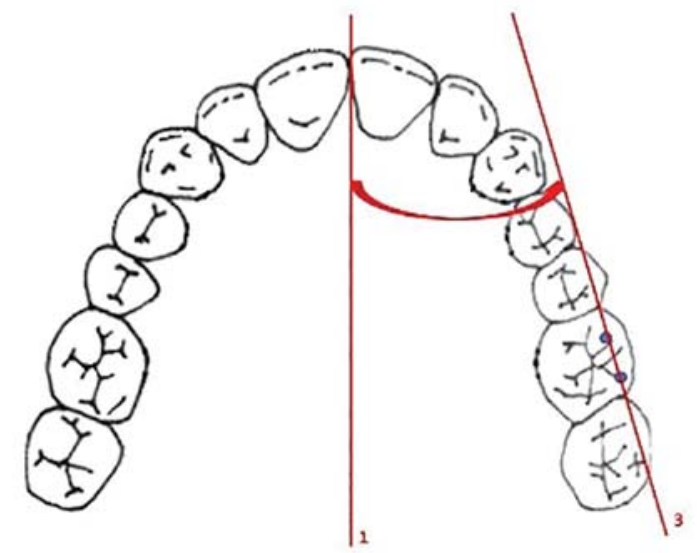

Fig. 3. Position of the upper first molars according to Henry

Cetlin and Ten Hoeve stated that in an ideal occlusion the buccal surfaces of the upper first permanent molars should be almost parallel to each other (13) (Fig. 4).

Variety of methods are discussed in the reviewed literature by different authors for the assessment of the upper molar rotation, which is valuable 


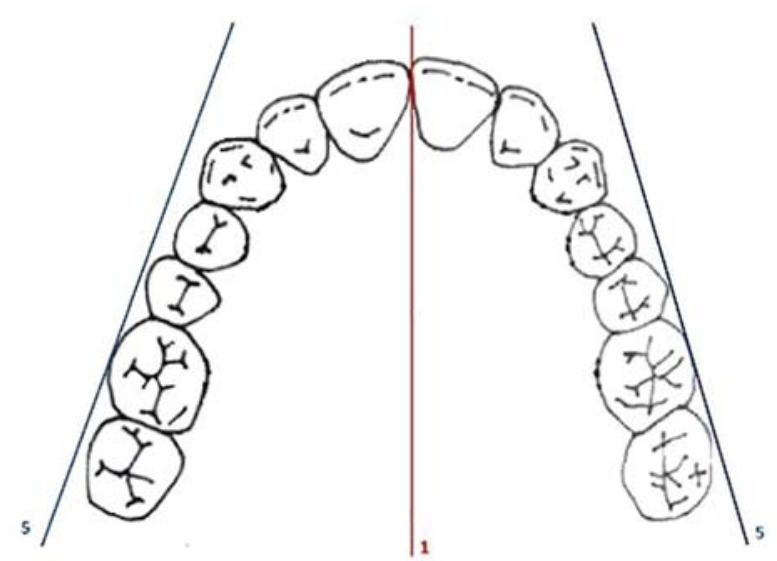

Fig. 4. Position of the upper first molars according to Cetlin and Ten Hoeve

in the early diagnostics and prophylaxis of the developing permanent dentition (12,14-19).

Vigano's method is the newest one for assessment of the upper first permanent molar rotation. According to his method (20) the rotation of upper first molars is measured by the angles formed by the intersection of lines going over the tips of the mesiopalatal and distobuccal cusps of each molar (Ricketts' line) with a straight line marked over the palatine raphe, as shown in Fig. 5 and Fig. 6. The standard magnitude of the angle is 67-73 degrees. Angles higher than 73 degree have an impact on the rotation of the molars. A deviation of 5 degrees is considered significant.

Rickett's guideline for clinical evaluation of the position of maxillary first molars on the occlusal view has been proposed using a line traced through the tips of the distobuccal and mesiopalatal cusps

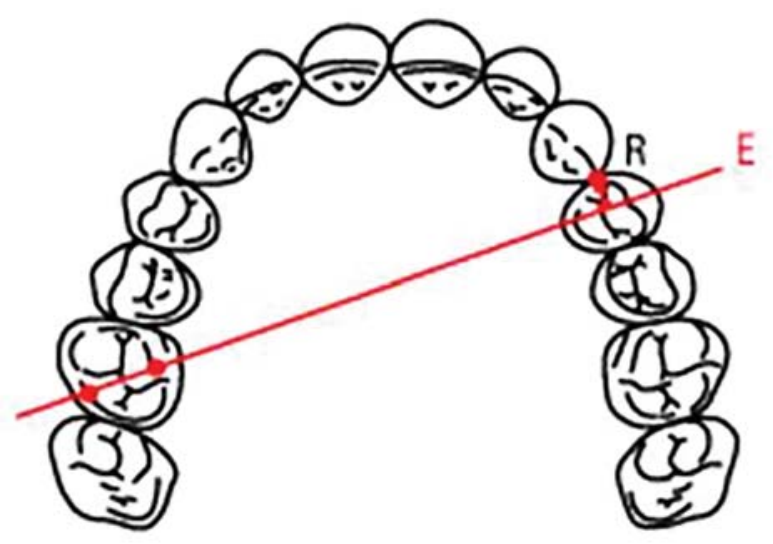

Fig. 5. Line of Ricketts

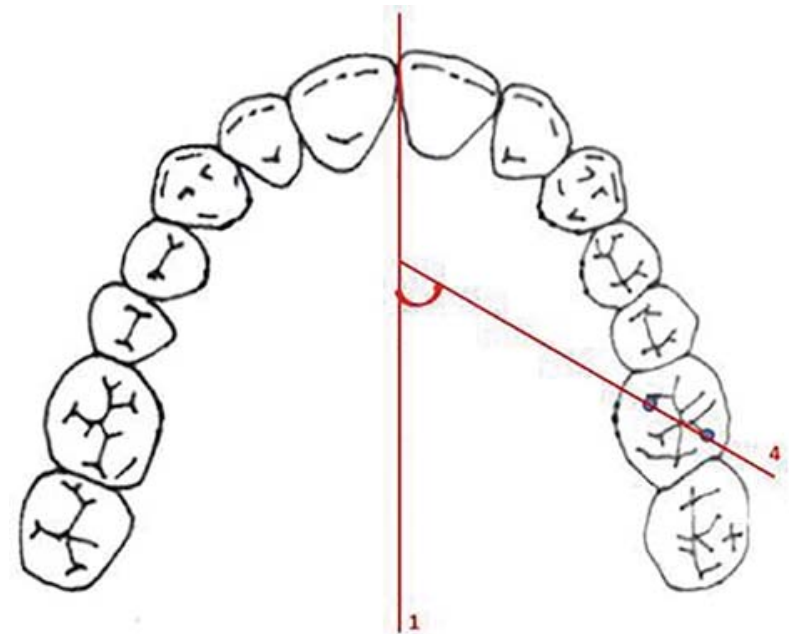

Fig. 6. Rotational position of upper first molar according to Vigano

of the permanent maxillary first molars. It was observed that, in normal occlusion, this line should pass through the distal third of the canine on the opposite side, and that this was a good guideline to analyze the mesiopalatal rotations, characteristic of mesial displacement of first molars in malocclusions.

It is preferable for more than one method for assessment of upper first permanent molar rotation to be used in order to reach an accurate diagnosis and treatment plan.

The mesiolingual rotation of upper first permanent molars is influenced by other factors such as: disturbances in tooth numbers - congenital absence of teeth - hypodontia, morphological shape and size of the molar and premolars, premature loss of primary teeth, interstitial caries of deciduous molars, dental arch shape (tapering, ovoid and squarios anterior arch forms), etc. $(21,22)$. Another factor could be transverse growth in the maxillary molar region at the time of the eruption of these teeth when the deciduous arch remains narrow $(23,24)$.

Maxillary first molars also rotate mesially because of mesial drifting of teeth as a result of attrition at the interproximal contacts of teeth due to an aging process.

This factors (variables) should be discussed and addressed when a specific method for assessment of molar rotation is planned or chosen.

The molars influence the transfer of occlusal forces to the facial skeleton. The upper first molar can tolerate more changes in the position than the lower 
one. The maxillary first molar is localized so that the mesiobuccal root continues into the infrazygomatic crest. When the molar is loaded with occlusal forces, the stress is transferred through the infrazygomatic crest. When mesial molar displacement occurs, the major part of the bite forces are transferred through the anterior part of the maxilla, resulting in the buccal bone receiving a compressive load. The shift of a single cusp width in the intramaxillary position of the molars in a mesiodistal direction has a significant impact on the occlusal load transfer (1).

The great majority of the cases with rotated upper first permanent molars or forward drift of the molar were associated with premature loss of deciduous second molars. Very often this is associated with rotation in a mesiolingual direction and a forward physical movement of the molars. This medial movement is simply the normal forward growth of the teeth, but proceeding in an uncontrolled manner because the contacts of the unbroken arch have been disturbed (6). The whole arch has been growing forward in accordance with normal growth processes. This opening of a path of lesser resistance may be a factor in the movement of molars after the extraction of their deciduous neighbors. If the upper permanent canine does not take up its position in the arch at the proper time, soon after the eruption of the premolars, the permanent molars take advantage of the extra room provided by the spacing of the premolars and rotate in a mesiolingual direction. The deciduous canine does not reserve sufficient space for its broader successor and the latter will be unable to find room in the arch.

It is a well-established fact that the permanent molars undergo progressive changes in their positions and in the direction of their movement during the course of development.

On a panoramic X-ray Kurol and Bjerklin (25) measured the axial inclination of the upper first molar. The tipping of the molars is measured by the angle formed between the tangent line to the mesial surfaces of the root and crown and the line through the lower margins of the left and right orbits. The normal value of the angle is $100^{\circ}$. A decreased value suggests mesial inclination of the maxillary first molar, which could lead to reduced arch length and crowding (Fig. 7).

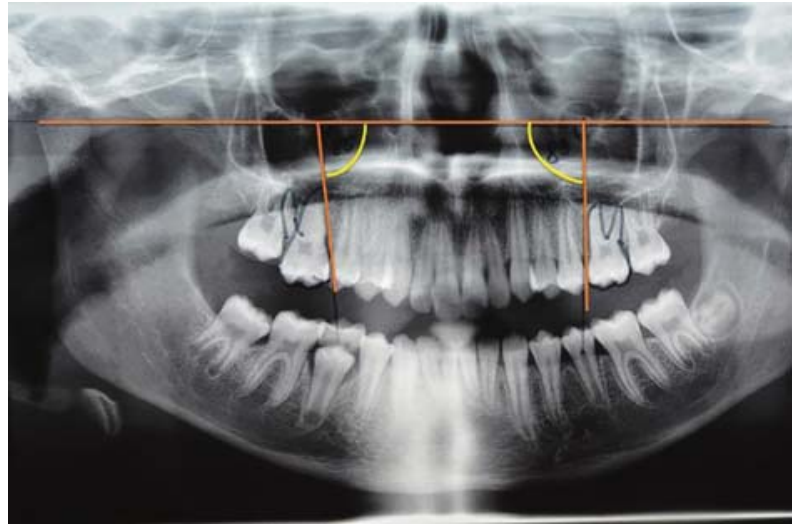

Fig. 7. Method of Kurol and Bjerklin

Atkinson (10), speaking of "a key ridge", has given us perhaps the clearest picture of the position of the first permanent molar in the maxilla. He states: "there is a definite relation of the buccal teeth to the key ridge all during life. The ridge is present at birth and remains in evidence even though the teeth are lost. At the age of 18 years, the roots of the upper first permanent molar wedge between the buccal and lingual walls of the maxillary bone and occupy a position in which the mesiobuccal root is directly under the key ridge. This is the most favorable position for the molars, from standpoint of function, mechanics, anatomy and facial harmony."

According to Sassouni's analysis (26) the mesial contour of upper first molar needs to lie on the $4^{\text {th }}$ arc - the temporal arc (Fig. 8). If the molar is anterior to this arc we could start a treatment with distalization. The temporal arc-nasion distance measured on the radius is equal to that from point ANS to the upper first molar. The position of the upper first molar varies with the position of the upper central in-

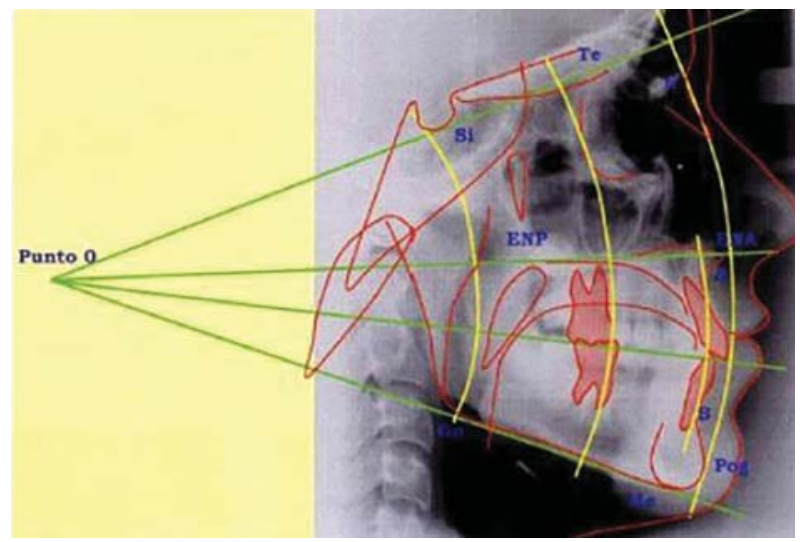

Fig. 8. Analysis of Sassouni 
The Importance of Upper First Permanent Molars Position for the Orthognatic Occlusion

cisors. The basic hypothesis is that if the upper first molar has a fixed position in the face, any increase in the total upper dental arch length will be transferred to the incisor area. Any change in the anteroposterior position of the upper first molar could influence the position of the mandibular leading to Class II malocclusion.

The axial inclination and rotation of the upper molar occur at the beginning of the transition of mixed to permanent dentition. Any changes in the sequence of primary teeth exfoliation have influence on dental arch length. Ricketts (19) pointed out that the average distance from the pterygoid vertical (PTV) plane to the distal surface of the upper first molar is the sum of the age of the patient $+3 \mathrm{~mm}$, in a growing patient. This diagnostic method is in aid of the orthodontist to decide whether to extract teeth or to distalize the molars (Fig. 9).

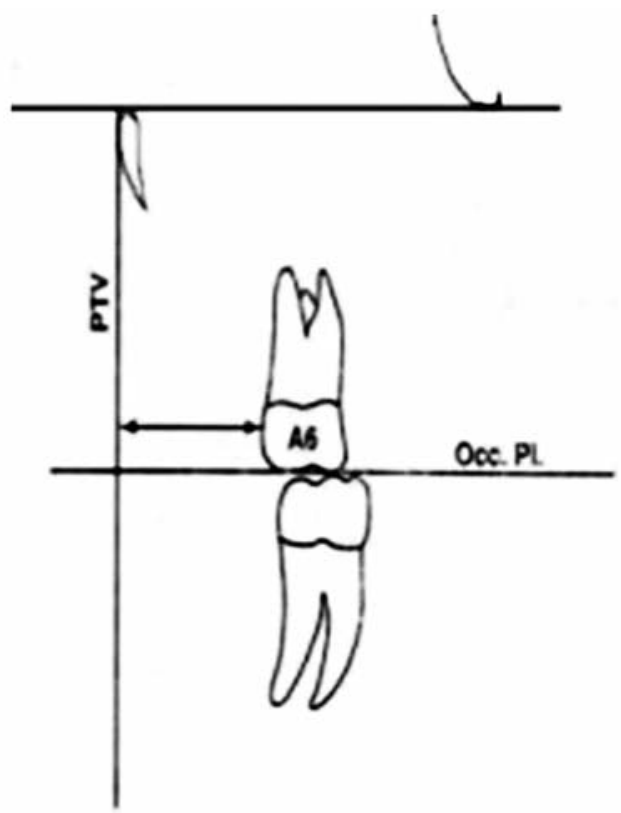

Fig. 9. Position of the upper first molar according to the analysis of Ricketts

Orientation and position of maxillary permanent first molar in the maxillary arch is very important in establishing molar relationship into Angle's Class I position.

Any loss of space in the arch is a justification for early orthodontic treatment (27). The problem of reduced arch length has an impact on the final treatment stage when the major orthodontic goal is estab- lishing of a tight teeth intercuspation between both arches. Mediopalatal rotation of the upper molar is an additional problem in the final phase as well.

\section{CONCLUSION}

The problem of reduced arch length has an impact on the final treatment stage when the major orthodontic goal is establishing of a tight teeth intercuspation. The molars influence the transfer of occlusal forces to the facial skeleton. Upper first molar tolerates more changes in the position than the lower one. The correct position of the upper molar ensure a stable occlusion with significantly low grade of relapse.

\section{REFERENCES}

1. Cattaneo PM, Dalstra M, Melsen B. The transfer of occlusal forces through the maxillary molars: a finite element study. Am J Orthod Dentofacial Orthop. 2003;123(4):367-73. doi: 10.1067/mod.2003.73.

2. Angle EH. Classification of malocclusion. Dental Cosmos. 1899;41:248-64,350-7.

3. Stoller AE. The normal position of the maxillary first permanent molar. Am J Orthod. 1954;40(4):259-71. doi: 10.1016/0002-9416(54)90089-5.

4. Andrews LF. The six keys to normal occlusion. Am J Orthod. 1972;62(3):296-309.

5. Strang RH. Textbook of Orthodontics. Blackwell Scientific Publication. 3rd Edition; 1950.

6. Smyth KC. The etiology and treatment of abnormally rotated molars. Int J Orthod Oral Surg Radiogr. 1931; 17(4):325-51. doi: 10.1016/ S0099-6963(31)80052-3.

7. Hellman M. An interpretation of Angle's classification of malocclusion of the teeth supported by evidence from comparative anatomy and evolution. Dent Cosmos. 1920; 62(4):476-95.

8. Lamons FF, Holmes CW. The problem of the rotated maxillary first permanent molar. Am J Orthod. 1961; 47(4):246-72. doi: 10.1016/0002-9416(61)90124-5.

9. Lima BP, Pinzan-Vercelino CR, Dias LS, Bramante FS, Tavarez RR. Correlation between the rotation of the first molars and the severity of class II division 1 malocclusion. ScientificWorldJournal. 2015;2015:261485. doi: 10.1155/2015/261485. 
Gergana Ivanova, Hristina Arnautska, Zornitsa Vulcheva et al.

10. Atkinson SR. The strategy of orthodontic treatment. J Am Dent Assoc Dent Cosmos. 1937; 24(4):560-74. doi: 10.14219/jada.archive.1937.0074.

11. McDonald JL, Shofer FS, Ghafari J. Effect of molar rotation on arch length. Clin Orthod Res. 2001;4(2):79-85.

12. Henry RG. Relationship of the maxillary first permanent molar in normal occlusion and malocclusion. Am J Orthod. 1956; 42(4):288-306. doi: 10.1016/0002-9416(56)90128-2.

13. Cetlin NM, Ten Hoeve A. Nonextraction treatment. J Clin Orthod. 1983; 17(6): 396-413

14. Friel S. Determination of the angle of rotation of the upper first molar to the median raphe of the palate in different types of malocclusion. Dent Pract. 1959;9:72-9.

15. Hansen GK, Caruso JM, West V, Andreiko CA, Farrage JR, Jeiroudi MT. The rotation of maxillary first molars, mandibular first molars, and maxillary first premolars in acceptable occlusions. Aust Orthod J. 1997;14(4):242-6.

16. Junqueira MHZ, Valle-Corotti KM, Garib DG, Vieira RB, Ferreira FV. Analysis of the rotational position of the maxillary first permanent molar in normal occlusion and Class II, division 1 malocclusion. Dental Press J Orthod. 2011;16(1):90-8. doi: 10.1590/S2176-94512011000100014

17. Naushad H, Shaheed S, Zahra S, Bano S. Maxillary first molar rotation and its relationship with skeletal and occlusal discrepancies. Pak Orthod J. 2014; 6(2):43-7.

18. Orton HS. An evaluation of five methods of derotating upper molar teeth. Dent Pract Dent Rec. 1966;16(7):279-86.

19. Ricketts RM. Occlusion-the medium of dentistry. J Prosthet Dent. 1969;21(1):39-60.

20. De Oliveira Viganó C, da Rocha VE, Junior LR, Paranhos LR, Ramos AL. Rotation of the upper first molar in Class I, II, and III patients. Eur J Dent. 2016; 10(1): 59-63. doi: 10.4103/1305-7456.175696.

21. Dale JG. Interceptive guidance of occlusion with emphasis on diagnosis. In: Graber TM, editor. Orthodontics: current principles and techniques. 2nd ed. Rio de Janeiro: Guanabara Koogan; 1996. p. 264-346.

22. Illingworth RS, Gardiner JH. Premature loss of deciduous teeth. Arch Dis Child. 1955;30(153):449-52.
23. Kim HK, Kwon HB, Hyun HK, Jung MH, Han SH, Park YS. Submorphotypes of the maxillary first molar and their effects on alignment and rotation. Am J Orthod Dentofacial Orthop. 2014;146(3):28693. doi: 10.1016/j.ajodo.2014.05.029.

24. Macena MCB, Tornisiello Katz CR, Heimer MV, de Oliveira e Silva JF, Costa LB. Space changes after premature loss of deciduous molars among Brazilian children. Am J Orthod Dentofacial Orthop. 2011; 140(6):771-8. doi: 10.1016/j.ajodo.2011.04.023.

25. Kurol J, Bjerklin K. Orthodontic syllabus for students. Sofia; 2014.

26. Sassouni V. Position of maxillary first permanent molar in the cephalofacial complex. Philadelphia. Am J Orthod. 1957; 43(7):477-510. doi: 10.1016/0002-9416(57)90175-6.

27. McNamara JA Jr, Brudon WL. Orthodontics and dentofacial orthopedics. Ann Arbor (Mich) : Needham Press; 2001. 